\title{
RESEARCH water fluoridation
}

\section{Correction: Erosion in children}

Unfortunately there was an editorial error in the letter from Michael Austin from Brighton, published in the BDJ 189: 526.

The section of the letter that read:

'Class 1 - Palatal surface of upper incisors and, or the incisal edges of these teeth eroded. Rounding palatally of the tip of the upper canine. NB enamel lesions only.

Class 2 - As above, plus enamel cupping of the mesiobuccal cusp of the lower first molars.

Class 3 - As above, plus cupping in enamel of all cusps of the lower first molars

Class 4 - As above, but lesions are present on the other teeth in addition eg upper first molars.

I have delibrately tried to keep the system as simple as is compatible with usefulness. The following quantifiers could also be used:

1. Half the area of enamel is lost from the occlusal surface of a tooth.

\section{All enamel is lost from a surface'}

Should have read:

'Class 1 - Palatal surface of upper incisors and, or the incisal edges of these teeth eroded. Rounding palatally of the tip of the upper canine. NB enamel lesions only.

Class 2 - As above, plus enamel cupping of the mesiobuccal cusp of the lower first molars.

Class 3 - As above, plus cupping in enamel of all cusps of the lower first molars

Class 4 - As above, but lesions are present on the other teeth in addition eg upper first molars.

I have delibrately tried to keep the system as simple as is comptible with usefulness. The following quantifiers could also be used:

++ half the area of enamel is lost from the occlusal surface of a tooth.

+++ all enamel is lost from a surface' 